\title{
SIMULAZIONE E DISSIMULAZIONE A SOSTEGNO DI POLITICA E AMORE SULLO SFONDO DELLA TRATTATISTICA DEL CINQUECENTO
}

\author{
Gennaro Valentino \\ Università di Stettino (Polonia) \\ gennaro.valentino@usz.edu.pl / ge.fides.valentino@gmail.com
}

Ricevuto: 28/04/2020 - Approvato: 26/05/2020 - Pubblicato: 30/07/2021

DOI: doi.org/10.17533/udea.lyl.n80a01

\begin{abstract}
Riassunto: Si propone l'analisi di due costituenti rinascimentali ben approfonditi nella precettistica e trattatistica del Cinquecento: la simulazione e dissimulazione. Attraverso lo studio di alcuni trattati precettistici, riflettiamo non solo sul fatto che nel Cinquecento queste pratiche comportamentali fossero riconducibili alla sfera dell'esteriorità, ma ragioneremo anche sul fatto che esse, mutuate dalla politica, si riversassero nella vita privata dell'individuo aiutandolo a raggiungere la «felicità amorosa», per dirla come Piccolomini. Si tenta di tracciare il fil rouge che collega gli scritti politici di Machiavelli e Guicciardini con La Raffaella, di Piccolomini, mettendo in luce gli obbiettivi conseguibili attraverso l'uso di tali pratiche comportamentali.
\end{abstract}

Parole chiave: Cinquecento; simulazione-dissimulazione; politica; amore; trattatistica.

\section{POLITICS AND LOVE UNDER SCHEMES OF SIMULATION AND DISSIMULATION ON THE BACKGROUND OF SIXTEENTH CENTURY TREATISES}

\begin{abstract}
The analysis of two Renaissance constituents is proposed, well-discussed during the sixteenth century: simulation and dissimulation. Through the study of some preceptistic works, there is a reflection not only about the sphere of exteriority of such behavioral practices, but also on their political origins and their insertion into the private life of the individuals, helping them to achieve «amorous happiness», as Piccolomini says. This is an attempt to trace the common thread between the political writings of Machiavelli and Guicciardini with La Raffaella, di Piccolomini, highlighting the achievable objectives through the practice of such habits.
\end{abstract}

Key words: sixteenth-century; simulation-dissimulation; politics; love; treatises.

Editores 


\section{Introduzione}

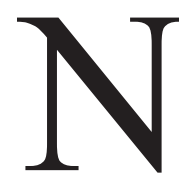

el presente articolo, attraverso lo studio di alcuni trattati ed opere precettistiche, riflettiamo non solo sul fatto che nel Cinquecento le pratiche comportamentali di simulazione e dissimulazione fossero riconducibili alla sfera dell'esteriorità — principalmente legate all'ambito politico e coltivate da coloro che ricoprivano ruoli di governo-, ma tenteremo di dimostrare come esse, mutuate dalla politica, si riversassero nella vita privata dell'individuo aiutandolo a raggiungere la «felicità amorosa» — per dirla come Piccolomini.

Nel dimostrare ciò, partiamo dai significati semanticamente più ampi dei termini «simulazione» $\mathrm{e}$ «dissimulazione», per poi passare a quelli che emergono dallo studio di alcuni scrittori, pensatori, nati e vissuti tra Cinque e Seicento. A seguire, ci concentriamo sul concetto di simulazione e dissimulazione in Machiavelli e in Guicciardini in riferimento all'ambito politico, ponendo il focus sul Segretario fiorentino e sulla teoria che era alla base della praxis politica, sottolineandone il suo allontanamento dalla morale tradizionale, nonché la sospensione del giudizio in merito all'uso di dette pratiche. Ciò ci permette di tracciare il fil rouge che collegherebbe gli scritti politici di Machiavelli, Il Principe, Discorsi, principalmente con La Raffaella di Alessandro Piccolomini, provando a far emergere l'influenza del fiorentino nei confronti del senese ${ }^{1}$. Nel paragrafo conclusivo si mettono in luce gli obbiettivi conseguibili sia in campo politico, che afferiscono alla sfera del pubblico, sia nel campo del sentimento amoroso, che al contrario afferiscono alla sfera del privato, grazie all'uso della simulazione e dissimulazione.

Bisogna ricordare che la proliferazione di questo tipo di opere è la conseguenza di chiare ed intense mutazioni sociali. Come spiega Alfano, «la letteratura comportamentale non può essere interpretata solo come 1'espressione di una volontà repressiva ed omologatrice, ma come l'indice delle tensioni che attraversano la società e la cultura italiane di quell'epoca» (Alfano, Gigante \& Russo, 2016, p. 262).

1. La bibliografia sul tema della simulazione e dissimulazione nei trattati del Cinquecento è molto ampia, si segnala: Lencan Stoica, G. \& Drāgulin S. (2014). New Studies on Machiavelli and Machiavellism: Approaches and Historiography. Bucarest: Ars Docendi. In essa si segnalano particolarmente: Cioalcā S. Le Prince et la Bête. La culture politique d'un notable florentin à la Renaissance. Quelques pistes méthodologiques.; Barbuto G. M. Le «Considerazioni intorno ai Discorsi sopra la prima Deca di Tito Livio» di Guicciardini; Minervini F. S. Aspetti seicenteschi della fictio tra politica e teatro; Suppa S. «Materia» politica e «forme» del potere in Machiavelli; Taranto D. Machiavelli e la «costruzione» della realtà. Ancora: Catanorchi O. (2005). Tra politica e passione. Simulazione e dissimulazione. En Leon Battista Alberti, Rinascimento, vol. 45, pp. 137-177; Senellart, M. (1997). Simuler et dissimuler: l'art machiavélien d'ètre secret à la Renaissance. En Historie et secret à la Renaissance, F. Laroque (Ed.). Paris : Presses de la Sorbonne Nouvelle, pp. 99-106.; Snyder J. R. (1994). Appunti sulla politica e l'estetica della dissimulazione tra Cinque e Seicento. Cheiron, vol. 11, n. 22, 23-43; Femia, J. (2014). Imparare a «poter essere non buono»: Machiavelli tra utilità e morale. En Biblioteca della libertà, XLIX n. 209; Fischer M. (1997). Machiavelli's Political Psychology. En The Review of Politics, 59, 4; Chabod, F. (1958). Machiavelli and the Renaissance (trad. D. Moore). New York: Harper and Row; Plastina, S. (2006). Su Piccolomini, si veda: Politica amorosa e «governo delle donne» nella Raffaella di Alessandro Piccolomini. En Bruniana \& Campanelliana: ricerche filosofiche e materiali storico-testuali, XII, 1; Piéjus, M. F. (1980). Venus bifrons: le double idéal féminin dans «La Raffaella» d'Alessandro Piccolomini. En Guidi, J., Piéjus, M. F. e Fiorato, A. C. (Eds.), Images de la femme dans la littérature italienne de la Renaissance. Préjugés misogynes et aspirations nouvelles. Paris : Université de la Sorbonne Nouvelle, pp. 81-167. 


\section{Simulazione e dissimulazione}

Se per simulazione si intende un atteggiamento che tende a far sorgere in altri un falso giudizio, per dissimulazione si fa riferimento ad una repressione volontaria d'ogni detto, atto o espressione mimica, che possa tradire pensieri, sentimenti, intenzioni. Dalla puntualizzazione appena marcata è possibile affermare, come è già ben noto, che i significati fissati dai dizionari odierni denotano le accezioni semantiche già contemplate all'epoca del Rinascimento.

Nel corso del XVI-XVII secolo, molti scrittori hanno approfondito con dovizia di particolari queste due pratiche comportamentali. Nel Libro del Cortegiano, in riferimento alla prudenza nella «elezion degli amici» (Castiglione, 1965, II, cap. XxIx, p. 130), Castiglione aveva fatto esporre a Bembo alcune riflessioni inerenti l'ambito dell'amore, poiché più volte era stato «ingannato da chi più amava e da chi sopra ogni altra persona aveva confidenzia d'esser amato» (Castiglione, 1965, II, cap. XXIX, p. 130). Nelle sue dichiarazioni, il cardinale umanista asserisce che soltanto la massima prudenza avrebbe dettato il giusto modo di comportarsi con gli altri, amici compresi, dei quali non era giusto fidarsi «per caro ed amato che sia», giacché «negli animi nostri son tante latebre e tanti recessi, che impossibile è che prudenzia umana possa conoscer quelle simulazioni, che dentro nascose vi sono» (Castiglione, 1965, II, cap. XXIX, pp. 130-131). Coloro che praticavano la dissimulazione erano considerati da Francesco Bacone uomini di opinabile affidabilità e insicuri delle proprie decisioni, in quanto «costoro sono costretti ad essere generalmente dissimulatori, come chi cammina con cautela perché non vede bene, mentre gli uomini più capaci sono stati sempre portati alla chiarezza e alla franchezza di comportamento» (Villari, 1987, p. 19). Di opinione netta era anche Giusto Lipsio, vissuto nella seconda metà del xVI secolo, che sosteneva l'inadeguatezza di queste pratiche comportamentali e di come dovessero essere «dalla vita humana bandite» (Lipsio, 1604, p. 145). Di contro, Torquato Accetto consiglia la pratica della dissimulazione all'uomo di sani principi per schermire quell'onestà da cui «non si forma il falso, ma si dà qualche riposo al vero, per dimostrarlo al tempo» (Accetto, 1997, p. 75), definendo quest'arte retorica come «un velo composto di tenebre oneste e di rispetti violenti» (Accetto, 1997, p. 75). Della stessa idea era anche Hugo Grotius, che considerava la simulazione vera e propria necessità purché adoperata con accortezza e prudenza.

\subsection{L'ambito politico}

La complessa analisi della simulazione e dissimulazione in riferimento alla sfera politica ci costringe a dare uno sguardo, seppur brevemente, alla letteratura latina di epoca repubblicana, accennando al De Catilinae coniuratione di Sallustio, in cui si descrive il comportamento e l'attitudine di Catilina: «Animus audax, subdolus, varius, cuius rei lubet simulator ac dissimulator, alieni adpetens, sui profusus, ardens in cupiditatibus; satis eloquentiae, sapientiae parum» (Sallusti, 2007, cap. v, par. 4). Questo passo è stato tradotto brillantemente da 
Alfieri: «di audace, ingannevole, versatile ingegno; d'ogni finzione e dissimulazione maestro; cupido dell'altrui; prodigo del suo; nei desideri bollente; più eloquente assai che assennato» (Alfieri, 1823, p. 10). Dalla narrazione che fa lo storico romano, emerge che, già ai tempi antichi, c'erano molte abilità che un uomo politico doveva possedere, tra cui alcune ascrivibili alle qualità fisiche, altre a caratteristiche esteriori: proprio tra queste ultime si posizionano su un gradino di rilievo simulazione e dissimulazione.

Passando al Trecento, è opportuno menzionare un personaggio della Divina Commedia, considerato il più grande ingannatore dell'opera, Guido da Montefeltro, fondamentale archetipo dantesco di dissimulazione politica. Nelle due terzine che seguono si mostra ciò che il Sommo poeta credeva del politico convertitosi poi al francescanesimo:

Mentre ch'io forma fui d'ossa e di polpe

che la madre mi diè, l'opere mie

non furon leonine, ma di volpe.

Li accorgimenti e le coperte vie

io seppi tutte, e sì menai lor arte, ch'al fine de la terra il suono uscie.

(Alighieri, 1966, Inf. XXVII, vv. 73-78)

Senza indugi teniamo a sottolineare che le opere di Guido da Montefeltro non furono praticate con la forza bruta, propria del leone, ma con scaltrezza e astuzia. Questa immagine rappresenta l'influenza che Dante aveva avuto su Machiavelli, che — come già è noto — passerà alla storia per le sue teorie politiche.

Nel Cinquecento, Francesco Guicciardini nei Ricordi fa riferimento ai consiglieri e uomini di governo facenti parte dell'entourage di Ferdinando il Cattolico, per i quali sarebbe stato opportuno esprimere le loro opinioni con lungimiranza. Lo scrittore conferma la necessità di usare l'arte della simulazione «solamente in qualche caso molto importante, le quali accaggiono rare volte» (Guicciardini, 1994, p. 51). L'uso parsimonioso di detta arte concederebbe la possibilità di essere creduto leale, libero, ma soprattutto di ricavarne maggiori benefici quando la metterà in pratica. Nell'esternare le proprie inclinazioni, Guicciardini precetta di dover simulare nei confronti di chi ascolta di avere «in animo el contrario, perché quando agli uomini pare che voi cognosciate che la ragione voglia così, facilmente si persuadono che le resoluzione vostre siano secondo quello che detta la ragione» (Guicciardini, 1994, p. 84).

Come accennato in precedenza, nel XVI secolo, l'ambito sociale per eccellenza in cui si necessitava l'applicazione di queste norme comportamentali era la politica. Machiavelli fa emergere come la simulazione sia al servizio della prassi politica e, dunque, l'importanza da parte del principe di attuarla. L'ormai celebre analisi in cui si consiglia al principe di dover sapere «usare la bestia e l'uomo», si fonda sulla necessità di governare sia attraverso le leggi che attraverso la forza, quest'ultima imprescindibile in politica poiché compensa le lacune lasciate dalle leges. Essendo inevitabile la connivenza di questa doppia natura, inoltre, il principe deve essere in grado di abitare i panni della bestia in una duplice forma, che Machiavelli spiega con la metafora della volpe e del leone. L'essere bestia non significa usare la forza esclusivamente in maniera bruta, ma anche furbescamente «perché il lione non 
si defende da' lacci, la volpe non si defende da' lupi. Bisogna adunque essere volpe a cognoscere i lacci, e lione a sbigottire i lupi» (Machiavelli, 1961, cap. XVIII, p. 64). In più, secondo il teorico fiorentino, l'uomo di governo che usa sia l'astuzia che la forza, incarnando la natura della bestia, è tenuto a «saperla ben colorire, ed essere gran simulatore e dissimulatore» (Machiavelli, 1961, cap. XVIII, p. 64). Dunque, si entra nel campo dell'apparenza, tesi che assume forza con le parole dello scrittore:

A uno principe, adunque, non è necessario avere in fatto tutte le soprascritte qualità, ma è bene necessario parere di averle. Anzi ardirò di dire questo, che, avendole e osservandole sempre sono dannose, e parendo di averle, sono utili, come parere pietoso, fedele, umano, intero, religioso, ed essere; ma stare in modo edificato con l'animo, che, bisognando non essere, tu possa e sappi mutare el contrario (Machiavelli, 1961, cap. XVIII, p. 65). ${ }^{2}$

In altri termini, per Machiavelli la cosa fondamentale è quella di fingere di possedere tutte queste qualità e di porle in atto solo quando «i venti e le variazioni della fortuna gli comandano». Sia Guicciardini che Machiavelli individuano con perspicacia i cambiamenti della società che vivono, che diviene la cifra comune da cui principiano le loro considerazioni politiche. Le caratteristiche che un principe deve possedere, finora segnalate, si ravvisano, in Ferdinando il Cattolico, esempio rintracciato anche nel Principe oltre che in Guicciardini, che «non predica mai altro che pace e fede», mentre in realtà «è inimicissimo» di entrambe. Come segnala Alessio Panichi, Machiavelli

sottolinea con forza la necessità politica della simulazione delle (buone) qualità e i suoi effetti benefici ai fini del mantenimento del potere, in conformità all'idea che tra essere, dover essere e apparire non possa e non debba esserci sempre una piena corrispondenza (Panichi, 2019, p. 18).

Altro esempio calzante è quello che il Segretario fiorentino fa nel Libro III dei Discorsi, menzionando la «simulata stultitia» ${ }^{3}$ di Bruto, il quale viene definito «mai tanto prudente, né tanto stimato savio» (Machiavelli, 1971, Libro III, cap. II, p. 265). In merito a questo passo del trattato, si rileva da parte dello scrittore una riflessione di riguardo e di encomio nei confronti del console romano, simbolo della lotta alla tirannia.

Dalla teoria politica di Machiavelli risulta come la prassi politica si basi, dunque, su un principio utilitaristico completamente slegato dalle categorie della morale tradizionale. A tutti gli effetti, il sembrare, parere, simulare, dissimulare, fingere, sono le armi riposte nella faretra di un principe che non gli permettono di agire su un piano ideale ma pratico, in contesti fattuali e anzitutto contingenti. In tal senso, si può certamente sostenere ciò che afferma Joseph Femia, ossia che il politologo del Cinquecento tentò di «separare la politica dalla morale tradizionale, in quanto le sue descrizioni del mondo intendevano basarsi sui fatti, sulle prove concrete, e non sulle esigenze di un sistema etico trascendente» (Femia, 2014, p. 39). Pertanto, l'esigenza della praticità, dettata dall'indiscutibile necessità politica, si coniuga alla pratica della simulazione e ne giustifica l'uso, poiché «Ognuno vede quel che tu pari; pochi sentono quel che tu sei» (Machiavelli, 1961, cap. XVIII, p. 66), il che fa risultare che sembrare sia più

\footnotetext{
2. I corsivi sono dell'autore. Il verbo parere è chiarificatore dell'idea esposta nel testo.

3. Si fa riferimento al Libro III, Capitolo II «Come egli è cosa sapientissima simulare in tempo la pazzia».
} 
importante che essere. In altri termini, i fattori segnalati: realtà sociale, necessità politica, esigenza della praticità, conducono il principe ad attuare comportamenti simulatori e dissimulatori, trascinando così la politica su terreni differenti dalla morale. Machiavelli non giudica i comportamenti di chi simula o dissimula, chi governa o chi è governato, ma teorizza una pura separazione: politica da una parte, morale dall'altra; la prima per essere proficua al massimo deve svincolarsi dalla seconda e rimanerne orfana. Tuttavia, sebbene ci sia una sospensione del giudizio, l'osservanza dei precetti teorici machiavelliani non può far altro che causare l'elusione dei tradizionali concetti di onestà e lealtà, cari all'amor cortese e al mondo feudale in genere.

\subsection{L'ambito amoroso}

Vorrei soffermarmi su quest'ultima riflessione, collegandomi ad Alessandro Piccolomini. L'allontanamento dalla morale tradizionale e la sospensione del giudizio sull'uso della simulazione e dissimulazione si ravvisano in Piccolomini nell'opera La Raffaella, ovvero dialogo della bella creanza delle donne. Il contesto culturale italiano in cui lo scritto viene ideato era molto complesso, il che è riscontrabile già solo in considerazione delle differenze culturali che affioravano dalle varie Accademie sparse un po' in tutta la penisola e che orientavano le produzioni letterarie degli scrittori che ne facevano parte.

È noto che il XVI secolo fu un periodo in cui emerse un'energica attività letteraria legata a questi centri culturali che si distinguevano gli uni dagli altri per i differenti campi di studio e produzione a cui si dedicavano, soprattutto per le spinte centraliste ed assolutistiche dei Signori che tendevano a controllare le aggregazioni degli intellettuali. In verità, furono due le teorie che accompagnarono lo sviluppo delle accademie: da una parte c'era chi faceva capo a Lorenzo Giacomini, che appoggiava il primato della libertà intellettuale; dall'altra c'erano gli speroniani che, invece, sostenevano il principio di autorità (Alfano, Gigante \& Russo, 2016, p. 117), poiché l'intellettuale senese credeva utile che un gruppo di studiosi dovesse essere ordinato ed organizzato e, dunque, ci fosse l'esigenza di un «principe tra li eguali» (Speroni, 1740, t. 3, p. 458). Oltre al contesto storico, è d'uopo far luce anche sul contesto personale in cui Alessandro Piccolomini va delineando i vari elementi del Dialogo, peraltro oggetto di commenti antitetici da parte di molti studiosi per quanto riguarda le motivazioni della sua genesi. Difatti, si è discusso se la natura dello scritto fosse filogina o misogina, in virtù di alcune dichiarazioni che lo stesso autore scrive nella lettera dedicatoria a De la Institutione, altra sua celeberrima opera, in cui espone a Laudomia Forteguerri ${ }^{4}$ 1'idea $^{\prime}$ di aver scritto l'opera «per un certo sollazzo, che per altra più grave cagione» (Piccolomini, 1543, p. III). Si dà

4. Su di lei: Eisenbichler, K. (2001) «Laodomia Forteguerri Loves Margaret of Austria», in Sautman, C. e Sheingorn, P. (a c. di) Same Sex Love and Desire among Women in the Middle Ages. New York: Palgrave, pagg. 277-304; Eisenbichler, K. (2003) «Poetesse senesi a metà Cinquecento: tra politica e passione», Rivista internazionale di letteratura italiana, pagg. 95-102; D’Amante, M. F. (2016) «Un trattato pedagogico del Cinquecento. L'Institutione di Alessandro Piccolomini», Educazione. Giornale di pedagogia critica, 5(1); Eisenbichler, K. (2012b) The Sword and the Pen. Notre Dame: University of Notre Dame Press; Robin, D. (2007) Publishing Women: Salons, the Presses, and the Counter-Reformation in Sixteenth-Century Italy. Chicago: The University of Chicago Press; Betri, M. L. e Brambilla, E. (2004) Salotti e ruolo femminile in Italia tra fine Seicento e primo Novecento. Venezia: Marsilio editore; Paoli, M. P. (1999) «Come se mi fosse sorella». Maria Selvaggia Borghini nella Repubblica delle lettere», in Zarri, G. (a c. di) Per lettera. La scrittura epistolare femminile in archivio e tipografia secoli XV-XVII. Roma: Viella, pagg. 491-534. 
nota di questo elemento per sottolineare il contesto in cui Piccolomini viveva al tempo, ossia a Padova tra gli Infiammati ${ }^{5}$, cosa che forse lo avrebbe portato a ritrattare la motivazione di partenza de La Raffaella, dal momento che l'Accademia padovana si attestava su linee politico-letterarie differenti da quelle adottate dall'Accademia senese. Gli studi inerenti la natura dello scritto piccolominiano lasciano tutt'oggi aperto il problema che sembra diventare sempre meno risolvibile per la sua complessità, tanto più se si considerano le altre opere dell'autore in cui si rintracciano, come già segnalato, dichiarazioni proprie che lo scagionerebbero dalle accuse di misoginia. ${ }^{6}$

L'opera di Piccolomini si inserisce in uno specifico contesto letterario connotato sia dalla tematica dell'amore sia dal genere del dialogo. Emerge una diversa visione dell'amore da parte degli umanisti e dei neoplatonisti ficiniani. Questi scelgono di servirsi di due differenti correnti di pensiero, una che si rifà a Platone, Plotino, Proclo ed ai platonici cristiani, l'altra fa riferimento alle tematiche della tradizione volgare della cultura fiorentina. La questione amorosa, tema caro a Piccolomini, è il vero leitmotiv di buona parte delle opere dei letterati a lui coevi, fatto che caratterizza La Raffaella anche per una nota di attualità che già al tempo le veniva riconosciuta.

Gli autori che contribuiscono alla formazione di questo contesto sono vari, come varie sono le opere che si possono menzionare. Quella più importante è certamente il De amore di Marsilio Ficino che viene dedicato a Cosimo de' Medici il Vecchio, suo mecenate, che lo appoggia anche per le sue traduzioni delle opere platoniche dal latino al greco. Il dialogo ficiniano è ambientato in una casa di campagna, a Careggi, regalo della famiglia medicea all'autore, che poi diventa l'Accademia Platonica. Gli attori di questo dialogo sono tutti amici dello scrittore. L'opera si apre il giorno 7 novembre che si pensava fosse il giorno di nascita e morte di Platone, ma anche la data in cui si svolgevano i Simposi, indetti dal mecenate Lorenzo de'Medici che voleva riportare in auge la tradizione platonica. Per Platone venne innalzato un altare con candele consacrate e ogni discorso veniva analizzato dai simposiasti. Il tema centrale delle conversazioni era l'amore, che veniva esaminato sotto vari punti di vista: mistico, medico, gnoseologico, cosmologico, astrologico e teologico. Il De Amore dal 1469 divenne il pilastro del platonismo rinascimentale divenendo un esempio per la lirica amorosa a lui successiva.

Un altro scrittore di rilievo che si aggiunge a Marsilio Ficino per il contributo dato al contesto letterario in cui Piccolomini agisce è Pietro Bembo con gli Asolani (1505) con cui legittima il modello ficiano. Quest'opera consta

5. Sull'Accademia degli Infiammati sorta a Padova nel 1540 cfr. Cerreta, F. (1960) A. P. letterato e filosofo senese del Cinquecento. Siena: Rizzoli, pagg. 19-41 e 263-278; Bruni F. (1967) «Sperone Speroni e l’Accademia degli Infiammati», in Filologia e letteratura, XIII/I, pagg. 24-71; Samuels R. S (1976) «Benedetto Varchi, the Accademia degli Infiammati, and the Origins of the Italian Academic Movement», in Renaissance Quarterly, xxIx, 4, pagg. 599-634; Daniele A. (1989) «Sperone Speroni, Bernardino Tomitano e l'Accademia degli Infiammati di Padova», in Filologia Veneta II, pagg. 1-53; Vianello, V. (1988) Il letterato, l'Accademia, il libro: contributi sulla cultura veneta del Cinquecento. Padova: Antenore; Baldi, A. (1991) «Alessandro Piccolomini e l'Accademia degli infiammati: Note in margine a un dibattito linguistico», Italian Culture, 9 (1), pagg. 115-127; Lo Re S. (1995-96), Benedetto Varchi. Contributi per una biografia, Tesi di dottorato in Storia moderna, Università degli studi di Catania. Assai ricca la bibliografia specifica su Pomponazzi e l'aristotelismo padovano; qui basti Nardi B. (1965) Saggi sul pensiero inedito di P. Pomponazzi, Firenze, Le Monnier; Kristeller P. O. (1983), Aristotelismo e sincretismo nel pensiero di Pomponazzi, Padova, Antenore; Kraye J. (2002), «La filosofia nelle università italiane del XVI secolo», in Le filosofie del Rinascimento, diretto da C. Vasoli, a cura di P.C. Pissavino, Milano, Mondadori, pagg. 350-373.

6. A tal proposito si segnalano gli studi di Marie Françoise Piéjus: (1980); e, in antitesi a quest'ultima, lo studio di Andrea Baldi: (2001). Inoltre, segnalo anche un mio lavoro sull'argomento: Valentino (2019). 
di tre libri sulla natura di Amore, in cui si individuano anche i presupposti filosofici della lirica petrarchesca? Vengono trattati due diversi contesti dell'amore, cioè il caso in cui si ama l'anima di una donna onesta, e l'altro in cui se ne ama solo il corpo. A Bembo «la discussione sull'amore gli consentiva di indicare la via da seguire per dar vita a un classicismo volgare che non fosse mera ripetizione di quello latino ma nascesse da una lucida interpretazione della cultura moderna» (Pozzi, 1980, Ix). Infatti negli Asolani:

l'inno all'amore spirituale e alla donna come tramite a Dio, con il quale il trattato si chiude, non deve far dimenticare i primi due libri. Perottino, Ghismondo e Lavinello in definitiva non presentano diverse posizioni filosofiche ma tre aspetti, contrastanti ma compresenti, dell'animo del Bembo e della lirica volgare; egli pertanto partecipa egualmente ai lamenti del primo, alle lodi di un amore più realistico e sensuale del secondo, come allo spiritualismo del terzo (Pozzi, 1980, x).

Gli Asolani furono considerati come il testo fondamentale del platonismo amoroso, soprattutto per la rappresentazione di Bembo fatta da Castiglione nel Cortigiano. Cronologicamente esso è il primo dialogo pubblicato che presenta donne come relatrici e dove la prefazione del terzo libro include una robusta difesa dell'inclusione di oratrici in una discussione filosofica, diretta contro i critici che pensano che loro siano incapaci di questi mansioni (Cox, 1992). Il dialogo di Bembo è ambientato in una vera e propria corte contemporanea (quella di Asolo, governata dalla deposta regina di Cipro, Caterina Cornaro), ma i suoi oratori non sono personaggi storici come nella tradizione ciceroniana, bensì sono finzioni poetiche identificate solo da nomi di fantasia. La stessa convenzione sarebbe stata adottata da numerosi scrittori di dialoghi successivi, come Agnolo Firenzuola e Moderata Fonte.

Pietro Bembo struttura 1'opera in tre parti: nella prima, parla Perottino e afferma che l'amore crea dolore e che è la più grande disgrazia sulla terra; nella seconda parte, Gismondo gli contrappone l'idea che l'amore è l'origine della felicità; infine, nell'ultima parte Lavinello si pone come mediatore tra i due uomini, effettuando una distinzione tra l'amore sensuale e l'amore spirituale, dove il grado massimo viene rappresentato dall'amore più bello. L'intreccio dei dialoghi prende le mosse dalla volontà della regina di Cipro di organizzare le nozze di una giovane donna, cornice in cui si muovono tre uomini che intrattengono discorsi sull'amore con tre donne sposate e normalmente frequentatrici di quei luoghi. L'assenza dei mariti delle tre è sintomatico del fatto che le donne sposate avessero più libertà delle nubili di frequentare luoghi in cui c'era la presenza di maschi. La conversazione non vede le tre donne come protagoniste; i loro interventi, come per il Cortegiano, si limitano a semplici brevi osservazioni.

Tramite il superamento dell'antitesi tra spirito e materia i trattatisti d'amore potevano rivalutare l'amore e

7. $\quad \mathrm{Al}$ De Amore possiamo segnalare altri testi analoghi: De amore (1508) di Francesco Cattani, il Libro de natura de amore (1525) di Mario Equicola, i Dialoghi d'amore (1535) di Leone Ebreo, il Ragionamento della Nanna e dell'Antonia e Dialogo nel quale la Nanna insegna alla Pippa di Aretino, incentrati su insegnamenti dell'arte puttanesca, il Dialogo d'amore (1535) di Speroni, il Dialogo amoroso (1543) e Il Raverta (1544) di Giuseppe Betussi, il Dialogo dell'infinità d'amore (1547) di Tullia d'Aragona, Specchio d'amore. Dialogo in cui alle giovani s'insegna innamorarsi (1547) di Bartolomeo Gottifreddi, Quesiti amorosi (1552) di Ortensio Lando nei Vari Componimenti (1552), A ragionar d'amore (c. 1554-55) di Marco Melechini, Aretefila (1557) di Luc'Antonio Ridolfi, Dialogo della lontananza (1563) di Lattanzio Benucci, L'amorosa filosofia (1577) di Petrić, Discorso sopra due questioni d'amore (1589) di Tasso. 
Simulazione e dissimulazione a sostegno di politica e amore sullo sfondo della trattatistica del Cinquecento

la donna. L'amore rimaneva un amore di bellezza, nella classica tradizione platonica e ficiniana, dove prese poi piede l'idea aristotelica della bellezza come armonia, unità e proporzione, cercando i canoni di quella corporea, individuandoli nei rapporti in cui stanno le misure delle singole parti. L'aristotelismo rivelava la povertà della bellezza nel momento della teorizzazione dell'amore.

Un altro trattatista fu Mario Equicola autore del Libro de natura de amore (1525). È trattato il tema dell'amore visto come una malattia giovanile da cui l'adulto doveva rinsavire. Circoscritto alla corte medicea, nonostante lui fosse veneziano, suscitò, per l'appunto, sconcerto e stupore nei critici del tempo. Nel suo dialogo vengono trattati i diversi fini e modi d'amare concedendo quattro amori all'uomo: 1'amore maritale, paterno, per l'amante e per sé stesso.

Leone Ebreo nei Dialoghi d'amore, pubblicati postumi nel 1535 a Roma, e concepiti e composti all'inizio del Cinquecento, approfondisce maggiormente il pensiero ficiniano. Egli si identifica in Filone e presenta, in particolar modo nel dialogo con Sofia, una scarsa argomentazione, un procedimento scolastico effettuato per dubbi, confutazioni, antitesi, con numerose citazioni del sapere greco, giudaico, gnostico e salomonico. L'opera, dallo stile e lingua imperfetti, come negli Asolani, presenta una struttura semplice: il primo libro definisce i concetti di amore e desiderio, il secondo presenta il problema della «comunità», ovvero della natura universale dell'amore, mentre il terzo, gli effetti dell'amore. Leone Ebreo si concentra su una sorta di amore cosmico, accattivandosi i lettori del tempo grazie anche alla civetteria di Sofia e la sua resistenza nei confronti di Filone, innamorato di lei. L'analisi dell'opera permette di estrapolare la concezione ficiniana dell'amore inteso come valore trascendentale. Garin evidenzia l'importanza di questa figura nella cultura rinascimentale e come erede della filologia umanistica attraversata da un senso morale (Garin, 1987).

Il Dialogo d'amore (1535) di Sperone Speroni presenta la poetessa e cortigiana Tullia d'Aragona in conversazione con Bernardo Tasso, raffigurato come suo amante, e una terza persona, Niccolò Grassi o Grazia. Per l'ideazione di una donna che parla in un dialogo filosofico sull'amore, Speroni potrebbe essere stato influenzato non solo dagli Asolani di Bembo, ma anche dal Simposio di Platone dove Socrate racconta la teoria dell'amore. La sua scelta di presentare Tullia d'Aragona nel ruolo di donna parlante suppone una grande novità. D’Aragona viene presentata in una situazione di intensità emotiva: è sul punto di separarsi da Tasso, che parte per Napoli, e il suo contributo alla discussione è appassionato e drammatico, sconvolgendo gli elevati stereotipi neoplatonici sull'amore spirituale che viene visto come strumento per superare la distanza fisica che li dividerà. Questo argomento verrà adottato dai suoi interlocutori che tentano di consolarla. Il suo ruolo non si limita a mera intervistatrice, ma ad esprimere opinioni, e il suo contributo al dialogo è importante, sia strutturalmente che filosoficamente, poiché aiuta a preparare il racconto materialista e aristotelico dell'amore. Secondo Deana Basile, Tullia viene designata come interlocutrice principale insieme a Grazia e viene messo Tasso in secondo piano, soffermandosi sulla capacità di azione della donna che si distanzia dalla figura della donna amata, vista come personaggio passivo del rapporto (Basile, 1999). Tullia specifica successivamente che il significato di «amare non è quello che suona il vocabulo, cioè fare e operare qualche cosa ma è più tosto un certo patir; e l'essere amato 
è verbo non passivo ma attivo» (Zonta, 1912, p. 546). Speroni, grazie allo status di cortigiana di d'Aragona, la caratterizza libera dalle rigide regole del decoro che governavano il linguaggio delle donne «rispettabili». Ma questa operazione la ripete anche nel suo Dialogo della dignità delle donne (1542) dove si esprimerà a lungo e con grande eloquenza la nobildonna padovana Beatrice Pio degli Obizzi, anche se parlerà in detrimento delle donne, sostenendo la loro inferiorità e la necessità di sottomissione delle mogli ai loro mariti.

Il Dialogo di amore, che univa le esigenze aristocratiche di Bembo e le preoccupazioni di modernità di Aretino, fu un modello di ispirazione per La Raffaella di Piccolomini, che rifletté una cultura vivace che la contraddistinse dal mondo universitario, lontana dalla struttura ragionativa e da ogni ostentazione di cultura. Speroni affermava due modi di filosofare e scrivere, quali «la maniera aristotelica, la quale conduce al sapere, e il dialogo platonico ben diverso da quello costruito per dimostrare una tesi e non privo di interventi diretti o indiretti dell'autore - che sente non poco della commedia» (Pozzi, 1980, p. XIX). Piccolomini segue questa sorta di dialogo filosofico dove vengono imitate le persone e le loro opinioni focalizzandosi sul diletto e sulla verità. Distinguerà i propri scritti dialogici dai dialoghi che non presentavano una conclusione, che avessero conclusioni discordanti o considerassero i dialoganti come personalità prive di intelletto e moralmente condannabili. I Dialoghi di Speroni rappresentano un nuovo genere letterario che unisce trattato e commedia, dove gli argomenti tipici della trattatistica vengono impiegati con comicità e sono un modello diretto di imitazione per Piccolomini ne La Raffaella.

Quando Piccolomini pubblica a Venezia il suo Dialogo (1539), sicuramente aveva letto anche le opere di Pietro Aretino, sempre attento alle preferenze dei lettori e del mercato editoriale. Il personaggio di Raffaella in parte è ispirato al Ragionamento della Nanna e della Antonia (1534) e, al Dialogo nel quale la Nanna insegna alla Pippa (1536), scritti riguardanti l'arte puttanesca, in cui la protagonista anziana tenta di impartire precetti alla giovane Pippa.

Tra le varie tematiche affrontate, quella amorosa si pone in primo piano nella Siena del Cinquecento e tra gli Intronati specialmente. La caratteristica principale è che si discute anche sul fronte parodico e filosofico, e quasi sempre attraverso il genere del trattato. Tra gli anni Trenta e Cinquanta del Cinquecento un grande numero di autori senesi o residenti a Siena si dedicano a questo genere letterario. Ad esempio, nel Dialogo sulla questione se sia meglio amare o essere amato di Girolamo Mandoli Piccolomini si discute di due lati di una quaestio d'amore, ossia se è meglio amare o essere amati. Il sermo pinceps lo ricopre una poetessa della scuola senese, Onorata Tancredi Pecci. La formula di quest'opera mostra due donne senesi che discutono con l'unico interlocutore maschile, Alfonso Todeschini Piccolomini, duca di Amalfi, che assume il ruolo più tipicamente femminile di ascoltatore e giudice (Doherty, 2008).

I casi che vedono due donne come sermones pincipes riguardano il dialogo senza titolo di Marcantonio Piccolomini pubblicato nel 1538 e quello di Marcello Landucci pubblicato nel 1542. Per il primo, questo ruolo è ricoperto da Laudomia Forteguerri che esprimere una serie di visioni religiose altamente speculative che vengono confutate dal più ortodosso Girolamo Carli Piccolomini, mentre per il secondo la figura di sermo princeps viene ricoperta da Atalanta Donati. Queste scelte confermano Siena come repubblica indipendente dove la partecipazione 
culturale delle donne è molto grande. Virginia Cox denomina questi dialoghi «quasi-documentari», poiché pretendono di raccontare una conversazione reale con figure identificabili di donne oratrici ${ }^{8}$ (Cox, 2013).

I dialoghi fino ad ora menzionati si distinguono per il loro carattere sperimentale e per l'importanza dei ruoli dati alle donne. I dialoghi di Marcantonio Piccolomini e Dell'economia overo del governo della casa (1555) di Aonio Paleario rappresentavano i primi esempi di dialoghi realisti che trascrivono conversazioni e che includevano esclusivamente donne oratrici, mentre il dialogo di Landucci offre un insolito esempio di una donna che parla nel ruolo di princeps sermonis alla presenza di un numeroso gruppo misto.

Quello esposto è il filone in cui si inserisce il Dialogo della bella creanza delle donne (1539) di Alessandro Piccolomini, dove anche il ruolo di sermo princeps è affidato a una donna, con la novità che questa non è né nobile, né famosa, né reale, ma piuttosto un prototipo di donna con esperienza nelle faccende amorose, identificabile con la figura della mezzana.

Il dialogo di Piccolomini avviene tra Raffaella, donna esperta e smaliziata, e Margherita, giovane donna di Siena. Raffaella tenta di convincere Margarita a commettere adulterio, tradendo il marito imprenditore sempre impegnato fuori città per lavoro, rompendo così il vincolo del matrimonio. La lunga conversazione si articola in un continuo sciorinamento di precetti impartiti alla ragazza, che toccano svariate tematiche: il senso pratico e morale dell'amore, l'attenzione da porre nel proprio aspetto fisico e la capacità nel porsi in pubblico, ma soprattutto 1'arte della simulazione e dissimulazione. ${ }^{9}$ Da subito, quindi, possiamo sottolineare come la pratica di questi due comportamenti non fosse precipuamente maschile — come appare dagli scritti politici sopra analizzati-, ma anche femminile. Pertanto, simulazione e dissimulazione risultano argomento comune alla trattatistica politica e alla precettistica indirizzata al genere femminile primo rinascimentale.

L'opera espone chiaramente l'obiettivo che si deve prefiggere la donna, per la quale è «convenevolissimo ch'ella con grande destrezza si ellegga uno amante unico in questo mondo e insieme con esso goda segretissimamente il fin de 1'amor suo» [Raffaella] (Piccolomini, 2001, p. 30). Alla giovane Margarita è consigliato di mettere in atto l'arte della simulazione, da sfruttare come strumento per raggiungere la felicità amorosa in segreto, e lontano dagli sguardi indiscreti di chiunque. L'avverbio «segretissimamente» rivelerebbe quanto il riconoscimento

8. Virgina Cox conta quasi quaranta «quasi-documentari» nel solo xvI secolo. Mentre alcuni di questi dialoghi sono ambientati a corte, come il Cortegiano di Castiglione, considerato il prototipo principale, gli altri sono ambientati in famiglie aristocratiche minori come quella della famiglia Beccaria a Pavia, scenario de La ghirlanda della contessa Angela Bianca Beccaria (1595) di Stefano Guazzo, o quella della famiglia Pallavicino a Cortemaggiore, vicino Piacenza, dove Luca Contile ambientò i suoi Dialoghi spirituali (1543).

9. In generale, i costituenti femminili rinascimentali possono essere rintracciati nelle opere di poeti, prosatori e filosofi di quel tempo che focalizzarono in parte la propria attenzione sulla figura femminile e sulle virtù muliebri, aspetto che dimostrava l'attualità della questione della donna già in quegli stessi anni, in varie occasioni e contesti. Ne sono un esempio i seguenti titoli: Angoscia doglia e pena di Michelangelo Biondo, Il libro della donna di Federico Luigini, Il convito overo del peso della moglie di Giovanni Battista Modio; e poi in maniera specifica riguardo la tematica amorosa, possiamo menzionare Ragionamento della Nanna e dell'Antonia e il Dialogo della Nanna scritti da Pietro Aretino, Specchio d'amore di Bartolomeo Gottifredi, Dialogo della signora Tullia d'Aragona della infinità d'amore di Tullia d'Aragona, Ragionamento di Sansovino, Il Raverta di Betussi Giuseppe. E poi, su tutti, il celeberrimo Cortegiano di Baldesar Castiglione, considerato radice e punto di partenza di svariate opere che lo seguono. Nell'opera castiglionesca si analizza la figura femminile specialmente nel terzo libro, ma è bene sottolineare che anche negli altri tre (come in diverse sue opere) si pone attenzione al genere muliebre, in comparazione al cortigiano. 
Simulazione e dissimulazione a sostegno di politica e amore sullo sfondo della trattatistica del Cinquecento

dell'indipendenza muliebre in seno alla libertà sessuale fosse al tempo inesistente. In ciò, però, Raffaella sottolinea l'unico modo per renderla possibile, ossia rivendicarla in maniera subdola e per l'appunto in segreto, senza il pretesto di farla diventare una componente femminile socialmente accettata. A questo proposito, è opportuno citare da La Raffaella un passo significativo che sottolinea la messa in opera della virtù della simulazione, in cui la ruffiana consiglia alla giovane di ricercarsi occasioni per mostrare la propria bellezza in pubblico, ma di farlo in modo assolutamente naturale:

e di tutto questo intendo che una giovene abbia da cercar destrissima occasione, e tale che non si pensi che ella abbia voluto che una tal cosa le intervenghi, perché in tutte le azioni od operazioni e parole di una donna, intendo principalmente che si abbia da conoscere con estrema onestà e pudicizia, perché, dove non è onesta, non s'apprezza né considera in una donna alcuna opera virtuosa; e per lo contrario, dov'ella è, altra cosa fiorisce. E però non solo ha da guardar ne le occasione ch'ella ha da pigliare, per far quanto ho detto di sopra, che altri non s'accorga che l'abbia fatto avertitamente, ma ha di finger con rossore, potendo arrossire con sua posta, o con qualche altro finto segno di onestà, d'aver avuto dispiacere che tal cosa le sia avvenuta (Piccolomini, 2001, p. 70).

In altri termini, il consiglio è di andare presso una sorgente d'acqua a rinfrescarsi ponendo in pubblico le proprie nudità, dove il «fingere con rossore» dissuaderebbe l'uomo dal credere che ci sia intenzionalità nell'attirare l'attenzione.

Se fino a questo momento l'arte del simulare è sfruttata per raggiungere la felicità amorosa, più avanti nel dialogo, nell'adozione di questo comportamento, si delinea una funzionalità strettamente materiale, ben diversa dalla precedente, come lascia intendere l'ennesimo consiglio dato da Raffaella:

una gentildonna [...] ha sempre in ogni sua azzione ed occorrenzia a mostrare, almeno fingendo, di avere desiderio di compiacere il marito suo in tutto quello ch'ella conosca che gli sia grado [...] e se non lo faccia con buon animo, almeno mostri di farlo, perché di qui nasce ch'ella può poi più arditamente spender ne le vesti: perochè, vedendola il marito così utile nel resto ed affezionata a la casa, non solo le compra questo volentieri, ma la esorta spesse volte a farlo (Piccolomini, 2001, pp. 77-78).

Dunque, praticare la simulazione sarebbe utile per indurre il marito all'acquisto di vestiti e oggetti di bellezza. Ne emerge, ancora una volta, l'immagine della donna come figura domestica e legata alle faccende di casa, la cui cura spronerebbe l'uomo a fare acquisti «volentieri» e «spesse volte», rimarcando la subordinazione totale alla figura maschile.

Pare interessante sottolineare anche un altro elemento importante, che potrebbe anche influire sulle opposte considerazioni, misoginia/filoginia, sul nostro autore. Giungendo al termine del dialogo, viene messo in luce come la simulazione/dissimulazione non sia una prerogativa femminile, ma condivisa da donne e uomini. Ad un'attenta analisi de La Raffaella, c'è da dire che l'arte della simulazione viene estesa anche al genere maschile. Come dichiara Raffaella, gli uomini «sanno fingere e piangere a loro posta e dimostrar d'essere tutto mèle, e poi riescono fiele e veleno» (Piccolomini, 2001, p. 99). Nel descrivere l'amante ideale per una donna, la mezzana si lascia sfuggire il nome di Aspasio, personaggio maschile dell'opera che non compare mai in scena e che pratica la simulazione, fingendo «qualche volta di amare alcune donne, non già per ingannarle, ma per ricoprire meglio 
per questa via lo amore ch'egli ha portato e porta a te» (Piccolomini, 2001, p. 116). Aspasio è l'uomo ideale per Margarita, giacché egli non solo la ama, ma al tempo stesso, simulando l'amore verso le altre donne, dissuade tutti dal far credere di provare amore per lei. Si parla, quindi, anche di riservatezza, che nell'atto del tradimento coniugale si configura come componente essenziale più volte rimarcata dalla mezzana nel corso del dialogo e si distingue come primo costituente della simulazione. Pertanto, la capacità di Aspasio di fingere non è considerata deprecabile, ma al contrario una virtù da elogiare, in quanto creerebbe condizioni fertili per il raggiungimento della loro felicità amorosa.

Inoltre, laddove in Guicciardini si segnalano parole di biasimo nei confronti di chi abusa della simulazione, scoraggiandoli a metterla in pratica senza giusta causa, ciò non avviene ne La Raffaella di Piccolomini dove, anzi, si rimarca più volte che «l'onore o il biasimo non consiste principalmente nel fare ella [la donna] una cosa o non la fare, ma nel credersi che la faccia, o non credersi» (Piccolomini, 2001, p. 84). Dunque, si evincono due consigli oggettivi per la precettata: primo, la possibilità di tradire il marito procurandosi un amante giovane e bello; secondo, nell'interesse verso se stessa e l'uomo tradito, mettere in pratica tutta la capacità di simulare l'amore, poiché «con li mariti basta a finger di amarli, e questo gli basta a loro» (Piccolomini, 2001, p. 102).

A questo punto, emergono due legami tra Machiavelli e Piccolomini. Primo: come già sottolineato nelle opere del Segretario fiorentino, anche nello scrittore senese osserviamo che gli esiti della simulazione, ossia tradire il marito e fingere di amarlo, si distanziano dagli atti morali tradizionali, che invece richiederebbero rispetto per il marito e del vincolo matrimoniale. Secondo: che anche per Piccolomini c'è una sospensione del giudizio nei confronti della moralità di Raffaella e Margarita. Lo scrittore non giudica né la giovane precettata, né la vecchia mezzana, tantomeno non sentenzia a favore di quei comportamenti, né critica i valori tradizionali.

Per quello che concerne più precisamente la dissimulazione, lungo il dialogo troviamo tra le due donne svariati richiami al non-detto, all'omesso, che principalmente si riferiscono ai tradimenti commessi dalla moglie. Se ne trova traccia nel seguente scambio:

\footnotetext{
RAFFAELLA

por cura che quei piaceri, i quali conchiuderemo oggi che ti si convenghino, tu venga di pigliarteli con tal ingegno e tal arte che il tuo marito più presto abbia da comportarlo volentieri che da pigliar un minimo sospettuzzo de casi tuoi. MARGARITA

In questo arrò io a durar poca fatica, perché, come vi ho detto, il mio marito è la miglior pasta d'uomo che voi vedeste mai. E di quelle cose che io mi disponessi, crederei farli credere che le lucciole fossino lanterne (Piccolomini, 2001, pp. 44-45).
}

Molto vicino a Piccolomini è Benedetto Varchi, il quale discute «Se l'amore si può simulare o dissimulare, e quale è più agevole di queste due cose» (Mauri, 1834, p. 226). Il suo trattato, Sopra alcune quistioni d'amore, ripropone la freddezza dell' autore filosofo che attraverso un linguaggio arido tenta di sciogliere dubbi su questioni amorose. Nella fattispecie, anche il Varchi individua l'arte della simulazione e dissimulazione come componente dell'amore, ma in maniera più netta, si schiera a favore della simulazione, affermando: 
Simulazione e dissimulazione a sostegno di politica e amore sullo sfondo della trattatistica del Cinquecento

Che uno, il quale non ami, simuli l'amore, cioè faccia le viste d'amare, pare a me, che non solo sia possibile, ma agevole e consueto in questi tempi. È bene, a giudizio mio, piuttosto impossibile che malagevole, dissimulare l'amore, cioè amando, far le viste di non essere innamorato. E come può essere, che alcuno ardendo tutto, non faccia alcun segno che egli abbrucia? (Mauri, 1834, p. 226).

Dal Varchi la dissimulazione viene quindi considerata «malagevole» e difficile da praticare poiché si rischierebbe di essere scoperti, trascinati dal fuoco dell'amore; mentre invece sarebbe opportuno simulare l'amore nei confronti dell'altro, azione considerata una pratica «possibile ed agevole». Questa opinione ricalca pienamente il precetto impartito anche dalla Raffaella di Piccolomini e sottolinea come la società vissuta da entrambi gli scrittori sia caratterizzata, per l'appunto, anche da questa prassi comportamentale, riscontrabile tanto negli uomini quanto nelle donne.

Ritornando a Piccolomini, si può menzionare un altro passo del Dialogo, in cui Raffaella mette maggiormente in luce quanto sia importante per la donna l'arte di simulare. Le parole della ruffiana ci ricordano il monito di Virgilio a Dante di fronte alla tomba del dannato Farinata degli Uberti, in cui la guida ricorderà al Sommo: «le parole tue sien conte» (Alighieri, Inf. X, 1321, v. 39). Il consiglio virgiliano viene ripreso da Raffaella che intima la giovane ragazza a «parlar poco e con accortezza» (Piccolomini, 2001, p. 79). Diversamente da ciò che l'intreccio del dialogo piccolominiano va definendo fino a quel momento, sembra quasi che la vecchia stia dando un consiglio moralmente valido alla giovane, esortandola a «non essere bugiarda né novellaia» (p. 79). Tuttavia, questo intento viene a cadere per le parole aggiunte in seguito che demoliscono l'apparente buona morale: «anzi mostri sempre il parlar puramente delle cose come sono, salvo quel che potesse nuocere a l'onestà sua; perché in questo è ragionevol di fuggire e simulare una cosa per un'altra più che si può» (p. 79). ${ }^{10}$ Qui il precetto sottolinea l'importanza di salvaguardare socialmente la propria immagine, atteggiamento mutuato dalla politica e dall'ambiente di corte, nel quale si intima di contrastare con ogni forza i giudizi altrui che minacciano la sua onestà, seppur rispondenti al vero. La bugia, dunque, diviene strumento per mettere in atto l'arte della simulazione. D'altronde, questo concetto sembra sovrapponibile a quello espresso dallo stesso Machiavelli nei confronti del principe, il quale deve avere

gran cura, che non gli esca mai di bocca una cosa che non sia piena delle soprascritte cinque qualità, e paia, a vederlo e udirlo, tutto pietà, tutto integrità, tutto umanità, tutto religione. E non è cosa più necessaria a parere d'avere, che quest'ultima qualità; perché gli uomini in universale giudicano più agli occhi che alle mani, perché tocca a vedere a ciascuno, a sentire a' pochi (Machiavelli, 1961, cap. XVIII, p. 66).

Dunque, sebbene con obbiettivi differenti, sembrerebbero emergere legami tra il comportamento che deve assumere un uomo di governo e quelli di una giovane donna, tra la teoria politica di Machiavelli e le idee di Piccolomini, soprattutto in seno alla sospensione del giudizio e alla separazione dalla morale tradizionale, segno di un concreto processo di mutuazione delle pratiche etologiche dalla politica alla sfera della felicità amorosa.

10. Il corsivo è dell'autore dell'articolo. 


\section{Conclusione}

Dalle opere selezionate ed analizzate, potremmo affermare che l'arte della simulazione e dissimulazione è praticata da donne e uomini indistintamente dal grado di estrazione sociale: uomini di rilievo politico e di comando e semplici civili, donne anziane o giovani.

I due ambiti analizzati, politica ed amore, ammettono l'uso della simulazione e dissimulazione per diversi scopi. Nel primo, gli obbiettivi da raggiungere afferiscono alla sfera del pubblico; difatti, il principe, il tiranno, l'uomo di governo, mettono in pratica queste due arti retoriche per il mantenimento del potere e il controllo della cosa pubblica. Nel secondo, gli scopi pertengono alla sfera del privato; difatti, il singolo individuo, come la Margherita di Piccolomini, nella propria intimità e contesto privato, simula e dissimula per il raggiungimento della felicità amorosa e l'appagamento sessuale, per rivendicare non tanto un libertinismo carnale, ma un'intraprendenza amorosa volta a sviluppare indipendenza sessuale libera dai pregiudizi di stampo misogino.

Da Piccolomini e Machiavelli emerge come simulazione e dissimulazione rappresentino un'essenziale caratteristica della politica e della felicità amorosa. Il senese non fa altro che sottolineare le radici della società in cui vive, pervasa da quei modelli tradizionali che si trascina dietro e che fatica ad estirpare, ed arroccata su un'idea di donna considerata come altro dell'uomo e sua appendice in aeternum. In ragione di ciò, secondo Raffaella, simulare e dissimulare sono le uniche vie d'uscita non solo per godere della propria bellezza e non reprimere la propria carica erotica, ma soprattutto per opporsi in sordina, senza contrastarle pubblicamente, alle idee pregiudizievoli dell'ordine costituito che ne offusca e definitivamente ne annulla la libertà sessuale. 


\section{Riferimenti bibliografici}

1. Accetto, T. (1997). Della dissimulazione onesta. S. S. Nigro (Ed.). Torino: Einaudi.

2. Alfano, G., Gigante, C., Russo, E. (2016): Il Rinascimento. Un'introduzione al Cinquecento letterario italiano. Roma: Salerno.

3. Alfieri, V. (1823). La guerra di Catilina. En Caio Sallustio Crispo tradotto da Vittorio Alfieri. Firenze: Ciardetti. 4. Alighieri, D. (1966-67). La Divina Commedia. En G. Petrocchi (Ed), Le opere di Dante Alighieri. Milano: A. Mondadori Editore.

5. Baldi, A. (2001). Tradizione e parodia in Alessandro Piccolomini. Lucca: Pacini Fazzi.

6. Basile, D. (1999) «Specchio delle rare e virtuose donne» : The Role of the Female Interlocutor in SixteenthCentury Dialogues on Love (Ph.D. Thesis). Toronto: University of Toronto.

7. Castiglione, B. (1965). Il libro del Cortegiano. G. Preti (Ed.). Torino: Einaudi.

8. Cox, V. (1992) The Renaissance Dialogue. Cambridge: Cambridge University Press.

9. Cox, V. (2013) The Female Voice in Italian Renaissance Dialogue. Italian Issue, 128(1), 53-78.

10. De Romieu, M. (1597). Instruction pour les jeunes dames, [tr. it. de C. Menna Scognamiglio, 1992]. Paris : Schena.

11. Doherty, P. A. (2008) «Arnica et non serva:» Love, Marriage, the Vernacular and the Querelle des Femmes in Six-teenth-Century Siena. London: London University.

12. Femia, J. (2014). Imparare a «poter essere non buono»: Machiavelli tra utilità e morale. En Biblioteca della libertà, XLIX n. 209. https://www.centroeinaudi.it/biblioteca-della-liberta/edizione-online/archivio-edizioneonline-categoria/174-anno-xlix,-n-209,-gennaio-aprile-2014/8935-imparare-a-\%C2\%ABpoter-essere-nonbuono $\% \mathrm{C} 2 \%$ BB-machiavelli-tra-utilit $\% \mathrm{C} 3 \% \mathrm{~A} 0$-e-morale.html.

13. Garin, E. (1987) Tra Medioevo e Rinascimento. Bari: Laterza.

14. Guicciardini, F. (1994). Ricordi. Milano: Masi.

15. Mauri, A. (1834). Opere di Benedetto Varchi, vol. 1. En Biblioteca enciclopedica italiana, vol. XxxvIII. Milano: Bettoni \& Comp.

16. Lipsio, G. (1604). Della politica overo dottrina civile libri VI. [tr. it. de Giovanni Martinelli, Roma]. 17. Machiavelli, N. (1961). Il Principe. L. Firpo (Ed.). Torino: Einaudi.

18. Machiavelli, N. (1971). I Discorsi sopra la prima deca di Tito Livio. En M. Martelli (Ed.), Tutte le opere. Firenze: Sansoni.

19. Ortensio, L. (1552) «Quesiti amorosi», in Varii componimenti di m. Ort. Lando. Vinegia: Gabriel Giolito de’ Ferrari e fratelli.

20. Panichi, A. (2019). «Bello è il mentir, se a far gran bene si truova». Verità, menzogna e simulazione negli scritti di Tommaso Campanella. En Lettere aperte, vi. «Testimoniare in versi», 9-23. 
Simulazione e dissimulazione a sostegno di politica e amore sullo sfondo della trattatistica del Cinquecento

21. Piéjus, M. F. (1980). Venus bifrons: le double idéal féminin dans «La Raffaella» d'Alessandro Piccolomini. En J. Guidi, M. F. Piéjus e A. C. Fiorato (Eds.), Images de la femme dans la littérature italienne de la Renaissance. Préjugés misogynes et aspirations nouvelles. Paris : Université de la Sorbonne Nouvelle, 81-167.

22. Piccolomini, A. (2001). La Raffaella, ovvero Dialogo della bella creanza delle donne. Roma: Salerno.

23. Piccolomini, A. (1543). De la Institutione di tutta la vita de l'huomo nato nobile e in città libera, Libri X in lingua toscana. Venezia: Scoto.

24. Sallusti G. C. (2007). Coniuratio Catilinae. I. Mariotti (Ed.). Bologna: Pàtron.

25. Pozzi, M. (1980) Trattati dell'amore del Cinquecento. Roma-Bari: Laterza.

26. Speroni, S. (1740). Opere di M. Sperone Speroni degli Alvarotti tratte da'mss. originali, 5 vol., III, Venezia: D. Occhi.

27. Tasso, T. (1589) «Discorso sopra due questione amorose», in Rime, et prose del S. Torq. Tasso. Venetia: Giulio Vasalini.

28. Valentino, G. (2019). Alessandro Piccolomini. La Raffaella. En Salvatore Bartolotta \& Mercedes Tormo-Ortiz (Eds.), Escritoras Italianas Inéditas en la Querella de las mujeres: traducciones en otros idiomas, perspectivas y balances. Vol. I. Madrid: Editorial UNED, 175-185.

29. Villari, R. (1987). Elogio della dissimulazione: la lotta politica nel Seicento. Roma-Bari: Laterza. 30. Zonta, G. (1912) Trattati d'amore del Cinquecento. Bari: Laterza. 